\title{
Hyperspectral darkfield microscopy of single hollow gold nanoparti- cles for biomedical applications
}

\author{
Natasha Fairbairn, ${ }^{a}$ Agathi Christofidou, ${ }^{a, b}$ Antonios G. Kanaras, ${ }^{a}$ Tracey A. Newman, ${ }^{b}$ and Otto L. \\ Muskens $^{* a}$
}

\author{
Received Xth $X X X X X X X X X X 20 X X$, Accepted Xth $X X X X X X X X X 20 X X$ \\ First published on the web Xth $X X X X X X X X X X 200 X$ \\ DOI: 10.1039/b000000x
}

Hyperspectral microscopy is a versatile method for simultaneous spatial and spectroscopic characterization of nonfluorescent samples. Here we present a hyperspectral darkfield imaging system for spectral imaging of single nanoparticles over an area of $150 \times 150 \mu \mathrm{m}^{2}$ and at illumination intensities compatible with live cell imaging. The capabilities of the system are demonstrated using correlated transmission electron microscopy and single-particle optical studies of colloidal hollow gold nanoparticles. The potential of the system for characterizing the interactions between nanoparticles and cells has also been demonstrated. In this case, the spectral information proves a useful improvement to standard darkfield imaging as it enables differentiation between light scattered from nanoparticles and light scattered from other sources in the cellular environment. The combination of low illumination power and fast integration times makes the system highly suitable for nanoparticle tracking and spectroscopy in live-cell experiments.

\section{Introduction}

Advances in nanoscience have resulted in the development of many new types of inorganic nanoparticles (NPs), which are finding use in a range of applications based on their exceptional chemical and physical properties. ${ }^{1-3}$ Amongst the wide range of inorganic NPs available, gold NPs have been used extensively in research, and are being developed as medical therapeutics. $^{4,5}$ Information about the interaction of gold NPs with biological tissue is important considering that gold NPs are already in the first stages of clinical trials (e.g. as part of a cancer treatment). ${ }^{6,7}$ The impact of the exposure to tissues and different organ systems of NPs has to be assessed. ${ }^{8}$ In particular, the nervous system is very likely to come in contact with NPs by multiple routes of delivery due to its spatial distribution within the body, and its close proximity to the NP exposure sites, for example skin. Therefore, there is an increasing need for understanding the interaction of inorganic NPs and neurons.

In recent years, a range of techniques has been developed and refined for imaging of nonfluorescent NPs at the singleparticle level. ${ }^{10}$ Darkfield microscopy, the detection of light that is elastically scattered by the NPs, is a powerful tool for

\footnotetext{
a Institute for Life Sciences and Faculty of Physical and Applied Sciences, University of Southampton, Highfield, SO17 1BJ, Southampton, United Kingdom. Fax: +44 238059 3910; Tel: +44 238059 3911; E-mail: O.Muskens@soton.ac.uk

${ }^{b}$ Institute for Life Sciences and Faculty of Medicine, University of Southampton, Highfield, SO17 1BJ, Southampton, United Kingdom.
}

imaging and spectroscopy of metallic NPs with sizes exceeding tens of nm. ${ }^{11-13}$ Darkfield spectroscopy, in combination with the high sensitivity of the localized surface plasmon resonances (LSPR) of metal NPs to small changes in the local environment, has been used extensively for sensing, ${ }^{14}$ with an accuracy approaching the single-molecule limit. ${ }^{15,16}$

While a broadband single-particle spectroscopic approach is suitable for high-resolution and/or fast imaging of individual NPs, it has several disadvantages. Importantly, the application of a broad spectrum of irradiation at once can produce a significant heating of the particle, which is especially unfavorable for in-vitro studies of sensitive biological systems. ${ }^{17,18}$ Further, applications involving NP counting and/or tracking favor a wide-field approach in combination with spectroscopic characterization. Various different approaches have been presented based on hyperspectral imaging where individual spectral components are filtered. ${ }^{19-21}$ Commercially available hyperspectral microscopy is capable of analyzing distributions of NP in biological systems. ${ }^{22,23}$ However, all of these techniques rely on spectral filtering of light collected from the sample, which does not solve the phototoxicity issue.

The development of high power collimated light sources based on supercontinuum fibre technology has enabled new opportunities for fast hyperspectral imaging. By combining a spectrally filtered source with a standard darkfield microscopy arrangement it is possible to perform spectral imaging over a large area with fast (millisecond) integration times. Here, we present a hyperspectral darkfield microscopy arrangement using an acousto-optic tuneable filter to sweep the illumination 
source over a spectral range from 500-1000 nm. The separation of spectral components at the illumination side has the additional advantage of allowing correction of chromatic abberations using a piezo focusing stage, which significantly extends the useable spectral range of the high-numerical aperture microscope objectives.

The applicability of the hyperspectral imaging system is demonstrated by performing spectral measurements on a selection of samples of hollow gold nanoparticles. Hollow gold particles are of particular interest for biomedical applications because their LSPR falls in the biological near IR window. ${ }^{18}$ Additionally biological molecules can be selectively encapsulated within and released from the hollow particle. ${ }^{24}$ In our current work, scattering spectra of colloidal hollow gold nanoparticles and particle clusters were measured and correlated with TEM images, and theoretical spectra were produced using finite element analysis to interpret the observed resonances. The hyperspectral imaging system was used to identify single nanoparticles that are taken up by SHSY-5Y cells after incubation of the cells for $1 \mathrm{hr}$ in the presence of nanoparticles, in order to demonstrate the potential of the system for cellular imaging applications.

\section{Experimental}

\subsection{Synthesis of hollow gold nanoparticles (NPs)}

Hollow gold NPs were prepared by first synthesizing spherical silver NPs and using them as templates in a galvanic replacement reaction. Initially, silver nanoparticles (seeds) were synthesised by silver nitrate $(2.5 \mathrm{mM}, 1 \mathrm{ml})$ reduction in the presence of trisodium citrate $(2.5 \mathrm{mM}, 1 \mathrm{ml})$, Milli-Q water $(8 \mathrm{ml})$ and ice-cold sodium borohydride $(0.001 \mathrm{M}, 25 \mu \mathrm{l})$. A growth solution was prepared containing Cetyl trimethylammonium bromide, (CTAB) $(0.2 \mathrm{M}, 80 \mathrm{ml})$, silver nitrate $(0.05 \mathrm{M}, 400 \mu \mathrm{l})$ and freshly prepared L-ascorbic acid $(0.1 \mathrm{M}$, $2 \mathrm{ml})$ at $35^{\circ} \mathrm{C}$. The silver seeds $(400 \mu \mathrm{l})$ were injected to the growth solution, followed by the addition of sodium hydroxide solution $(1 \mathrm{M}, 2 \mathrm{ml})$. A colour transition was observed from colourless to orange-yellow, signifying the formation of silver NPs (templates for the formation of hollow gold particles). The reaction was completed after 24 hours and the final product was purified from excess CTAB by centrifugation/decantation (2500 rcf, $15 \mathrm{~min}$ ) and redispersed in Milli-Q water $(40 \mathrm{ml})$. The hollow gold NPs were formed by a slow, constant addition of sodium tetrachloroaurate (III) dihydrate aqueous solution $(1 \mathrm{mM}, 0.25 \mathrm{ml})$ to a silver nanoparticle solution $(10 \mathrm{ml})$, which was heated to reflux. There was a colour transition from orange-yellow to deep blue, indicating the formation of hollow gold NPs. The NP solution was left to cool down to room temperature and was stored at $4{ }^{\circ} \mathrm{C}$. Figure 1 shows a Transmission Electron Microscopy (TEM) image of

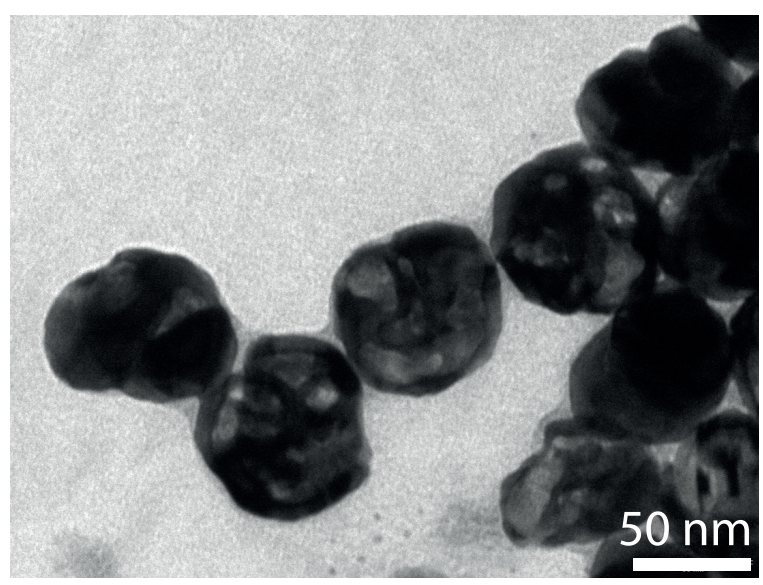

Fig. 1 Transmission Electron Microscopy (TEM) image of CTAB-coated hollow gold nanoparticles.

the CTAB-coated hollow gold nanoparticles.

Hollow gold NPs were capped with mono-carboxy (1mercaptoundec-11-yl) hexaethylene glycol (OEG-COOH), to ensure colloidal stability and allow further functionalisation of the NPs (e.g. with peptides) via the $\mathrm{COOH}$ end of the ligand. In detail, a fresh OEG-COOH solution $(5 \mathrm{mg} / \mathrm{ml}, 200 \mu \mathrm{l})$ was prepared and injected into a colloidal solution of hollow gold NPs $(0.5$ O.D, $5 \mathrm{ml})$ while sonicating at $4{ }^{\circ} \mathrm{C}$ for one hour. Then, the NP solution was stirred for 30 minutes at room temperature. The reaction mixture was kept overnight at $4{ }^{\circ} \mathrm{C}$ and was finally purified from the excess ligand by three centrifugation/decantation steps (8000 rpm, $15 \mathrm{~min}$ ). NPs were redispersed in sodium borate buffer $(0.1 \mathrm{M}, \mathrm{pH} 9)$ and kept for futher use.

A neuron targeting peptide (Tet1- KHLNILSTLWKYR) was conjugated to the OEG-capped gold NPs in the presence of (1-(3-(dimethylamino)propyl)3-ethyl-carbodiimidemethiodide (EDC) and $\mathrm{N}$ hydroxysulfosuccinimide (s-NHS) coupling agents. Namely, the peptide ( $100 \mu \mathrm{l}, 0.15 \mathrm{mM}$; $0.1 \mathrm{M}$ sodium borate buffer $\mathrm{pH}$ 9) was added to $5 \mathrm{ml}$ of gold NPs (0.3 O.D.; $0.1 \mathrm{M}$ sodium borate buffer $\mathrm{pH} 9$ ), followed by the addition of EDC; $50 \mu 1,0.2 \mathrm{M}$ ) and s-NHS; $100 \mu 1,0.2 \mathrm{M}$ ), and shaken gently overnight at room temperature. The peptide-OEG-NPs were purified from excess peptide ligands and coupling reagents by three purification steps $(13,400 \mathrm{rpm}, 15$ minutes per step), and redispersed in $100 \mu$ l of cell growth media.

\subsection{Cell preparation}

SH-SY5Y cells were maintained in F-12 cell media which was supplemented with penicillin/streptomycin, non-essential amino acids and 5\% fetal bovine serum, in a humidified atmosphere of $5 \% \mathrm{CO}_{2}$ and $95 \%$ air, at $37{ }^{\circ} \mathrm{C}$. They were seeded 


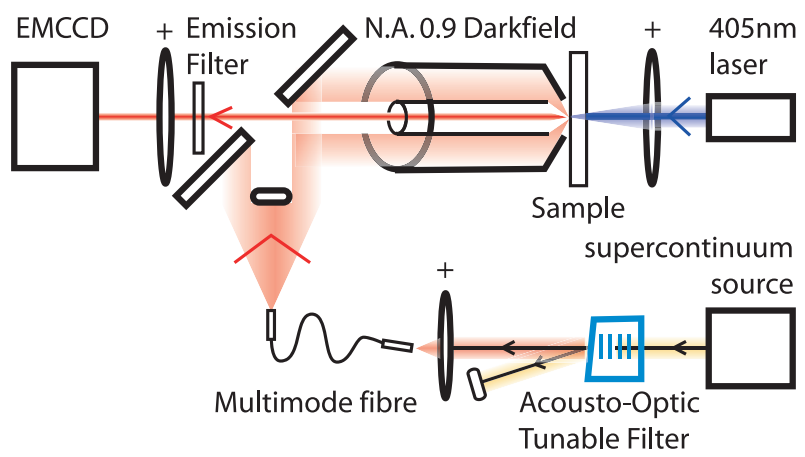

Fig. 2 Experimental Setup for hyperspectral darkfield microscopy using a supercontinuum fibre light source and acousto-optic tuneable filter in the illumination.

in a 12-well plate on glass coverslips, at a density of $2 \times 104$ cells per well, in $1 \mathrm{ml}$ supplemented cell media. The following day, cells were differentiated by the addition of retinoic acid (1 $\mu 1,3 \mu \mathrm{g} / \mathrm{ml})$.

SH-SY5Y cells were incubated with the peptide-OEG-NPs for $1 \mathrm{hr}$ (1 O.D.), and then washed three times with PBS (x1, $\mathrm{pH}$ 7.4). The samples were fixed by 15 minute incubation with $4 \%$ paraformaldehyde. All reagents were pre-warmed at $37{ }^{\circ} \mathrm{C}$, to achieve good fixation of the samples. The fixative was then removed by washing the samples three times with PBS, followed by water. The glass coverslips were washed again with plenty of Milli-Q water (excess water was blotted off) and then mounted with 4', 6-diamidino-2-phenylindole (DAPI, a fluorescent dye which stains the cell nuclei) mounting media onto a glass microscope slide.

\subsection{Hyperspectral darkfield microscope}

The experimental setup is shown schematically in Fig. 2. A supercontinuum light source (Fianium SC400-2) produced a collimated beam of white light with a total power of $2 \mathrm{~W}$ and a spectral power density of several $\mathrm{mW} / \mathrm{nm}$. A co-linear acousto-optic tuneable filter (Crystal Technology) was driven by a USB-controlled RF generator/amplifier (Isomet) to spectrally filter the broadband source over an octave-spanning range from $450 \mathrm{~nm}$ to $>1000 \mathrm{~nm}$, with a bandwidth of around $1 \%$ of the center wavelength. The diffracted light from the AOTF was focused into a multimode optical fibre. By tilting the fibre to an angle of around $20^{\circ}$, it was possible to couple into a high-order mode producing a donut-shaped angular profile at the output. The diverging angular donut of light was collimated and sent into the 0.95 N.A. reflective illumination ring of the microscope objective (Nikon CFI LU Plan BD $100 \times, 0.9$ N.A.). The transmitted optical power of $0.1 \mathrm{~mW}$ at $700 \mathrm{~nm}$ was illuminating an area of around $150 \mu \mathrm{m}$ in diameter, resulting in relatively low illumination intensities of
$<0.5 \mathrm{~W} / \mathrm{cm}^{2}$. Light scattered from the sample (ie. at the same wavelength as the illumination light) was collected by the refractive central part of the objective and was imaged onto an EMCCD camera (Andor Luca R). No EM-gain was used for the measurements of this work. The sample was mounted on a $3 \mathrm{D}$ piezo stage to enable precise adjustments of position and focus.

Hyperspectral images were collected using a Labview program which controlled both the AOTF and the EMCCD camera. Between each wavelength step, a manual correction of the image for chromatic aberrations was done using the 3D piezo stage by optimizing the image of a bright reference particle. Further image processing was done using Matlab. Areas of interest were selected from the image, roughly corresponding to the diffraction-limited spots of individual nanoparticles. Intensity spectra were extracted from the datacube from these areas, including a tracking algorithm which corrected for small residual image drift. The spectra were corrected for spectral response of source, camera, and optics by using a spectrum taken from a calibration sample. The calibration was done using a layer of strongly scattering white paint $\left(\mathrm{TiO}_{2}\right)$ which produced a uniform and wavelength-independent, diffuse Lambertian scattering pattern. The resulting calibrated intensity scale corresponds to 1000 EMCCD counts for a value of 1.0 at $630 \mathrm{~nm}$ wavelength. In addition to darkfield scattering, fluorescence images from cells stained with DAPI were obtained using excitation by a 405-nm laser diode and a 430-nm longpass emission filter in the detection beam path (see Fig. 2).

\subsection{Correlated TEM analysis}

For the purpose of comparing single nanoparticle spectra with transmission electron microscopy (TEM) images, hollow gold nanoshells were deposited onto copper TEM finder grids, which had previously been coated with formvar and carbon. TEM images were taken using a transmission electron microscope (FEI Tecnai 12) at $120 \mathrm{kV}$.

\subsection{Finite element analysis}

The optical response of hollow gold nanoparticles was modeled using Comsol Multiphysics 4.2a. All models used a spherical volume with a radius of $250 \mathrm{~nm}$ and $30 \mathrm{~nm}$ thick PMLs. The sizes and separation of nanoparticles were estimated from the TEM images. The shell thickness of the nanoparticles was estimated by comparing the theoretical spectra to the experimental data. The Johnson and Christy data was used for the dielectric response of gold. ${ }^{25}$ The host refractive index was modeled using a simple effective medium approximation based on the mean of the refractive index of air and that of the formvar coating used on the TEM-grids, resulting in an effective refractive index of 1.22. The theoretical 
a.
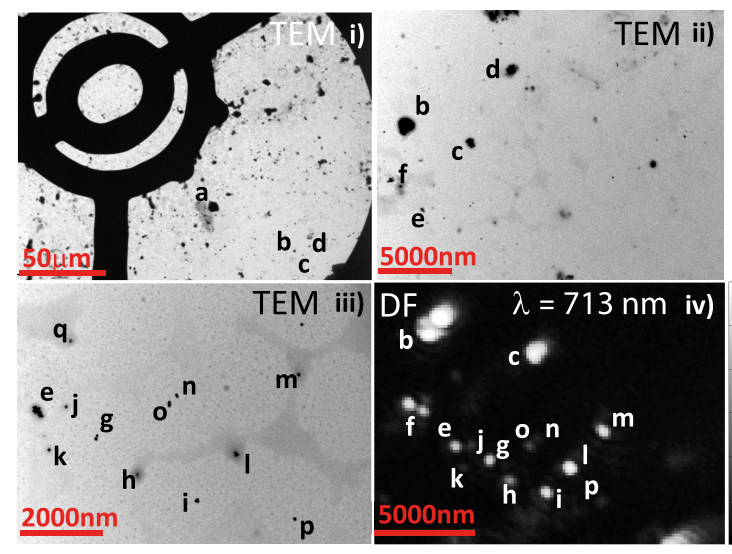

C.
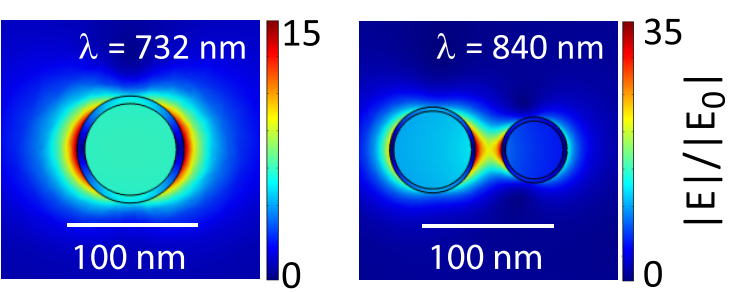
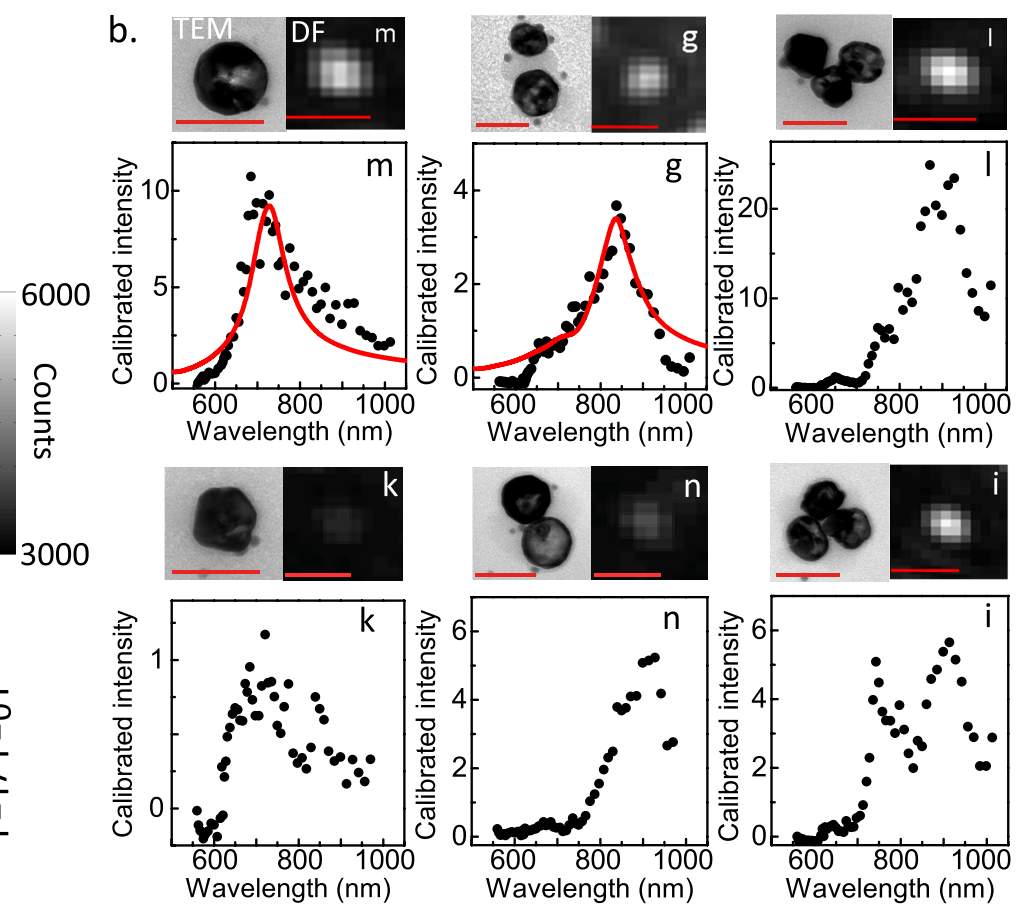

Fig. 3 a. TEM images i)-iii) of nanoparticles on a marked finder grid, individual objects are labeled $a-p$. iv) Darkfield image at $713 \mathrm{~nm}$ wavelength of the corresponding area. b. Spectra of objects $m, k, g, n, l$ and $i$ obtained from hyperspectral images (integration time: $0.2 \mathrm{~s}$ per point), shown along with the corresponding TEM and darkfield $(\lambda=713 \mathrm{~nm})$ images of the nanoparticle. Scale bars: $100 \mathrm{~nm}$ for TEM and $1 \mu$ for darkfield images. Red lines: numerical model calculations of spherical hollow gold NP with optimized shell thickness. c. Calculated near-field maps of the local field enhancement $\left|E / E_{0}\right|$ for the models of single particle $m$ and dimer $g$.

scattering spectra were produced by performing a surface integral of the scattered electric field over a spherical shell surrounding the nanoparticles and taking the absolute value of this parameter. The theoretical spectra were scaled vertically to match the experimental intensity.

\section{Results and Discussion}

\subsection{Hyperspectral Measurements of Hollow Gold Nanoparticles}

To test the sensitivity of the setup in detecting singlenanoparticles, we performed a correlated TEM and optical study using OEG-coated hollow gold NPs deposited onto a marked TEM-grid. Figure 3 shows three TEM images at different magnifications and one image taken with the darkfield microscope with incident light at a wavelength of $713 \mathrm{~nm}$. The letters a to $\mathrm{p}$ correspond to clusters of nanoparticles identified in the images. Spectra of selected individual objects are shown in Fig. 3b, along with close up TEM and darkfield images of the objects. Objects $m, k$ correspond to single hollow gold NPs, while objects $g, n$ are NP dimers, and $l, i$ are NP trimers. Overall variations in the optical intensity are observed which are thought to originate from variations in the focus alignment of each image. Significant corrections of the focal distance of up to $20 \mu \mathrm{m}$ were necessary to correct for chromatic abberations at wavelengths longer than $700 \mathrm{~nm}$, due to the limitations of the achromatic darkfield objective. Despite these corrections, reproducible results could be achieved within $20 \%$ of the intensity, as was found by repeated hyperspectral scans of the same NPs (not shown).

As can be seen Fig. 3b, the single hollow gold NP $m$ shows a resonance at $685 \mathrm{~nm} \pm 10 \mathrm{~nm}$. This resonance is in agreement with other single-particle studies on hollow gold ${ }^{27}$ or $\mathrm{Au} / \mathrm{Au}_{2} \mathrm{~S}$ nanoshells. ${ }^{26}$ We have qualitatively reproduced the spectral response (red line) using a numerical model for a hollow gold nanoparticle with a diameter of $83 \mathrm{~nm}$ and a shell thickness of $5.5 \mathrm{~nm}$. The NP diameter was obtained from the TEM image, however the shell thickness was optimized to achieve agreement with the spectral resonance position. Detailed TEM images such as Fig. 1 show that a large variation of shell thickness exists between different NP and that the thickness varies even within a single hollow gold NP. In addition, the exact morphology deviates from a perfect sphere for most particle, which further complicates quantitative analysis of the spectral response. The mode profile corresponding to the peak 
of the spectral resonance of particle $m$ is shown in Fig. 3c. The near-field map shows that the resonance can be attributed to a dipolar mode of the hollow gold NP, with a local field enhancement of one order of magnitude. In comparison to $m$, particle $k$ shows a much weaker response. The difference in absolute intensity between the two particles reflects the difference in particle size as observed from the TEM images. We recall that, for small NP, the scattering cross-section scales with the square of the particle volume, which means that small variations in size can result in large differences in detected intensity. The measurement of NP $k$ shows that the setup has the sensitivity to detect individual nanoparticles with sizes below $50 \mathrm{~nm}$ with good signal to noise and relatively short integration time $(0.2 \mathrm{~s})$.

In comparison to the single nanoparticles, the dimer and trimer clusters show a redshifted spectral response, in agreement with the expectation for strongly interacting nanoshells. ${ }^{26}$ The theoretical spectrum shown for NP $g$ in figure $3 \mathrm{~b}$ was produced by modeling two nanoparticles with diameters of $60.5 \mathrm{~nm}$ and $79 \mathrm{~nm}$ and a shell thickness of $3.7 \mathrm{~nm}$, with a separation distance of $24 \mathrm{~nm}$. This separation was relatively large compared to the other clusters $n, i, l$. As can be seen more clearly in Fig. 1, some agglomeration of particles occurs on the TEM grid, resulting in very narrow gaps and concomitantly large spectral redshifts due to NP interactions. The trimer cluster $i$ shows an even more complex response of multiple resonances, possibly due to plasmonic Fano-resonances caused by the interference of the different sized NP in the trimer. ${ }^{28}$

\subsection{Hyperspectral Measurements of SHSY-5Y Cells In- cubated with Hollow Gold Nanoparticles}

We demonstrate the potential of our hyperspectral darkfield microscope in studying the interactions of single nanoparticles with mammalian cells, such as the cells of the central nervous system. The uptake of hollow gold NPs by SHSY-5Y cells was used as a model system for imaging. SHSY-5Y cells have been incubated for $1 \mathrm{hr}$ with hollow gold nanoparticles coated with Tet1 peptide. The cells were imaged using brightfield microscopy and DAPI fluorescence, and the resulting information was overlayed with the darkfield scattering image at $713 \mathrm{~nm}$ to identify the positions of nanoparticles relative to the cells. Figure 4 shows the results for a SHSY-5Y incubated with NPs coated with Tet1 peptide. A clear contribution of darkfield intensity can be identified in the spatial map which is associated with the position of the cell. Individual bright spots were selected for further spectroscopic analysis, as shown in spectra $\mathrm{i}$ - iv of Fig. 4. The spectra of i, iii, and iv show a peak around 700nm which is consistent with the approximate spectral resonance and intensity of individual hollow gold NPs as established from our correlative TEM study. In addition,
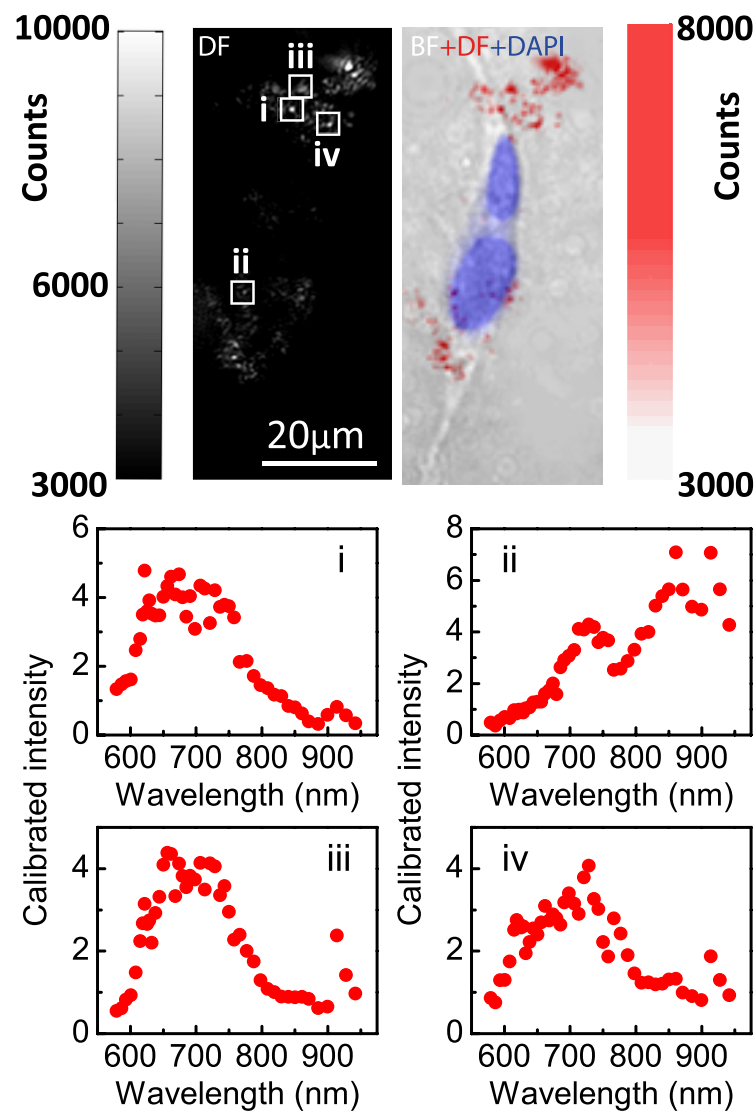

3000

Fig. 4 (a) Darkfield image at $713 \mathrm{~nm}$ and (b) a combined false colour image showing darkfield, DAPI fluorescence and brightfield images of a SHSY-5Y cell that has been incubated with hollow gold nanoparticles for $1 \mathrm{hr}$ are shown along with spectra $\mathrm{i}$ - iv, taken from different bright spots indicated on (a). Integration time: $0.1 \mathrm{~s}$ per image.

the spectrum of position ii shows a more complex redshifted response, which is consistent with the typical response for a cluster of two or more NPs. The amount of nanoparticles inside the cell and their degree of clustering depends strongly on the type of NP coating. ${ }^{29}$ Generally, polyelectrolyte-capped NPs show less nonspecific uptake, whereas the Tet1 peptide specifically binds to the trisialoganglioside clostridial toxin receptor on neurons, possibly promoting a receptor-mediated endocytosis. ${ }^{30}$

Figure 5 shows images of a cell from a control sample of SHSY-5Y cells, which was not incubated with hollow-gold NPs. Again, intensity is observed when the cell is viewed in darkfield. Overall the intensity is lower and smooth over the entire cell, without sharp features. We attribute this intensity to light that has been scattered from the cell. This contribution can be clearly differentiated from the nanoparticle signals by examining the measured spectra i-iv. The spectra measured 


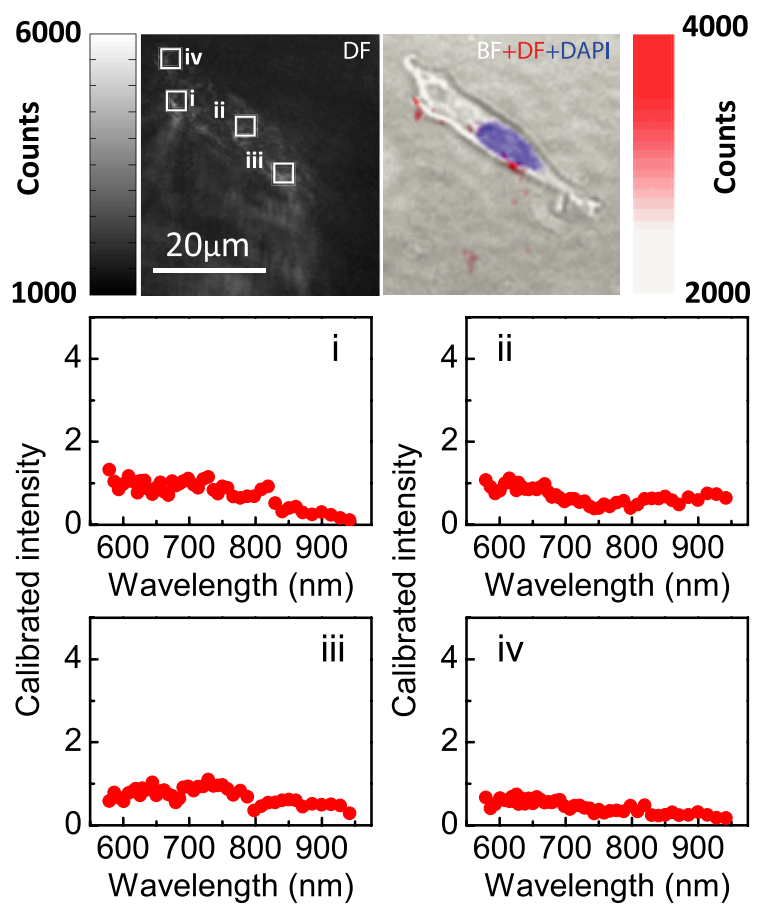

Fig. 5 (a) Darkfield image at $713 \mathrm{~nm}$ and (b) a combined false colour image showing darkfield, DAPI fluorescence and brightfield images of a control SHSY-5Y cell that has not been incubated with hollow gold nanoparticles are shown along with spectra i - iv, taken from different bright spots indicated on (a). Integration time: $0.1 \mathrm{~s}$ per image.

for the control cell do not show the characteristic peaks of nanoparticle resonances and are about 5 times lower than the signals obtained from the individual NPs.

Applications of metal NPs in biomedicine cover a wide range from laser hyperthermia to plasmonic biosensing. Therefore, sensitive techniques capable of assessing their interactions with living cells are of importance. Compared to nonfluorescent scattering NPs, luminescent quantum dots generally have advantages related to imaging applications and long-term cell tracking, especially in combination with multiphoton and confocal detection. ${ }^{31,32}$ Metal NPs can be functionalized with additional fluorescent labels, however cofunctionalization of several moieties on the surface increases complexity and may affect the biophysicochemical properties. Metal NPs are stable and do not photobleach, allowing for long-term tracking. Thus, while our hyperspectral darkfield microscope cannot compete with confocal fluorescence in terms of sensitivity, it opens up new opportunities for the monitoring of nonfluorescent particles.

A promising next step will be the implementation of realtime spectral sensing using the plasmonic (LSPR) response of gold NPs. Here, our method will have a potential advantage over other hyperspectral techniques ${ }^{19-23}$ as we can strategically select a number of wavelengths around the LSPR. This in principle allows fast readout of the LSPR of a large number of NPs, e.g. in biological systems or in microarray type devices. The feasibility of this approach for real-time parallel sensing will be explored in future studies.

\section{Conclusions}

We have demonstrated a hyperspectral darkfield microscope using a scanned supercontinuum light source. The setup has been successful for imaging and spectroscopy of individual gold nanoparticles. Comparison with TEM showed the capability of measuring individual hollow gold NP as small as $50 \mathrm{~nm}$ with a short integration time per image of $0.2 \mathrm{~s}$. The system also allows identification of these individual hollow gold NP in fixed cell preparations, at illumination intensities $<0.5 \mathrm{~W} / \mathrm{cm}^{2}$. Such intensities have been shown to result in minimal toxicity for cells incubated with metal NPs ${ }^{18}$. This advantage of using a scanned illumination source will be important for future live cell studies of nanoparticle uptake and processing in cells, where phototoxicity is currently a limitation. Such a capability will enable new investigations of NP uptake and transport, and the use of single NP as spectral sensors of specific sub-cellular activity ${ }^{21,33}$.

\section{References}

1 Xie, J., Liu, G., Eden, H. S., Ai, H., and Chen, X. Acc. Chem. Res., 2011, 44, 883-892.

2 Erathodiyil, N. and Ying, J. Y. Acc. Chem. Res., 2011, 44, 925-935.

3 Saha, K., Agasti, S. S., Kim, C., Li, X., and Rotello, V. M. Chem. Rev., 2012, 112, 2739-2779

4 Arvizo, R. R., Bhattacharyya, S., Kudgus, R. A., Giri, K., Bhattacharya, R., Mukherjee, P., Chem Soc Rev., 2012 41, 2943-2970

5 Dreaden, E. C., Alkilany, A. M., Huang, X., Murphy, C. J., and El-Sayed, M. A., Chem. Soc. Rev., 2012, 41, 2740-2779

6 Alkilany, A. M., Lohse, S. E., Murphy C. J., Acc Chem Res. 2012, ASAP

7 Barreto, J. A., O’Malley, W., Kubeil, M., Graham, B., Stephan, H., Spiccia, L., Advanced Materials 2011, 23, H18-H40.

8 Zhao, F., Zhao, Y., Liu, Y., Chang, X., Chen, C., Zhao, Y., Small 2011 7, 1322-1337.

9 Murray, W. A. and Barnes, W. L., Adv. Mater. 2007, 19, 3771-3782.

10 Van Dijk, M. A., Tchebotareva, A. L., Orrit, M., Lippitz, M., Berciaud, S., Lasne, D., Cognet, L. and Lounis, B., Phys. Chem. Chem. Phys., 2006,8, 3486-3495.

11 Mock, J, Barbic, M., Smith, D. R., Schultz, D. A. and Schultz, S. J. Chem. Phys. 2002116 6756-6759.

12 Sonnichsen, C., Reinhard, B. M., Liphardt, J. and Alivisatos, A. P. Nat. Biotechnol. 200523 741-745.

13 Urban, A. S., Pfeiffer, T., Fedoruk, M., Lutich, A. A., Feldmann, J. ACS Nano 2011 5, 3585-3590.

14 Anker, J. N., Hall, W. P., Lyandres, O., Shah, N. C., Zhao, J. and Van Duyne, R. P. Nat. Mater. 2008, 7, 442-453.

15 Acimovic, S.S., Kreuzer, M. P., González, M. U., and Quidant R., ACS Nano 2009 3(5) 
16 Ament, I., Prasad, J., Henkel, A., Schmachtel S, Sönnichsen, C., Nano Lett. 2012, 12, 1092-1095.

17 Krpetic, Z., Nativo, P., Sée, V., Prior, I. A., Brust, M. and Volk, M., Nano Lett 2010, 10, 45494554.

18 Bartczak, D., Muskens, O. L., Nitti, S., Sanchez-Elsner, T., Millar, T. M and Kanaras, A. G. Small 2012 8, 122-130.

19 Becker, J., Schubert, O., and Sönnichsen, C., Nano Lett. 2007, 7, 1664 1669.

20 Bingham, J. M., Willets, K. A., Shah, N. C., Andrews, D. Q. and Van Duyne, R. P., J. Phys. Chem. C, 2009, 113, 1683916842.

21 Zhang, L., Li, Y., Li, D.-W., Jing, C., Chen, X, Lv, M., Huang, Q., Long, J.-T., and Willner, I., Angew. Chem. Int. Ed., 2011, 50, 67896792.

22 Hutter, E., Boridy, S., Labrecque, S., Lalancette-Hébert, M., Kriz, J. Winnik, F. M. and Maysinger, D. ACS Nano, 2010, 4, 2595-2606.

23 Grabinski C., Schaeublin, N., Wijaya, A., DCouto, H., Baxamusa, S. H., Hamad-Schifferli, K., and Hussain, S. M. ACS Nano, 2011 5, 2870-2879.

24 Wu G., Mikhailovsky A., Khant H. A., Fu C., Chiu W., Zasadzinski J. A., J. Am. Chem. Soc. 2008, 130, 8175-8177.

25 Johnson, P. and Christy, R. Phys. Rev. B 1972, 6, 4370-4379.
26 Lassiter, J. B., Aizpurua, J., Hernandez, L. I., Brandl, D. W., Romero, I., La, S., Hafner, J. H., Nordlander, P., and Halas N. J., Nano Lett., 2008, 8, 12121218.

27 Schwartzberg, A. M., Olson, T. Y., Talley, C. E., and Zhang, J. Z. J. Phys. Chem. B 2006, 110, 19935-19944.

28 Brown, L. V., Sobhani, H., Lassiter, J. B., Nordlander, P., Halas, N. J. ACS Nano, 2010, 4, 819832.

29 Rosman, C., Pierrat, S., Henkel, A., Tarantola, M., Schneider, D., Sunnick, E., Janshoff, A., and Sönnichsen, C. Small, 2012, DOI: 10.1002/smll.201200853.

30 Zhang, Y., Zhang W., Johnston, A. H., Newman, T. A., Pyykkö, I., Zou, J., Int. J. Nanomedicine 2012, 7, 1015-1022.

31 Parak, W. J., Boudreau, R., Le Gros, M., Gerion, D., Zanchet, D., Micheel, C. M., Williams, S. C., Alivisatos, A. P., Larabell, C. A, $A d v$ Mater. 2002, 14, 882-885.

32 Michalet, X., Pinaud, F. F., Bentolila, L. A., Tsay, J. M., Doose, S., Li, J. J., Sundaresan, G., Wu, A. M., Gambhir, S. S., Weiss, S., Science, 2005, 307, 538-544.

33 Jun, Y., Sheikholeslamia, S., Hostetter, D. R., Tajon, C., Craik, C. S., and Alivisatos, A. P. Proc. Nat. Acad. Sc., 2009, 106, 17735-17740. 